\title{
Cryogenic and large-base Fabry-Perot cavities for ultra-stable laser systems
}

\author{
Denis S. Kryuchkov ${ }^{1, *}$, N.O. Zhadnov ${ }^{1}$, K.S. Kudeyarov ${ }^{1}$, I.A. Semerikov ${ }^{1}$, \\ K.Yu. Khabarova ${ }^{1}$, and N.N. Kolachevskiy ${ }^{1,2}$ \\ ${ }^{1}$ P. N. Lebedev Physical Institute of the RAS, Moscow 119991, Russia \\ ${ }^{2}$ Russian Quantum Center, Skolkovo, Moscow 143025, Russia
}

Nowadays most of high-precision spectroscopy experiments are based on lasers with spectral linewidth less than $1 \mathrm{~Hz}$. Best systems now provide fractional frequency instability $4 \times 10^{-17}$ on 1-100 seconds averaging time [1]. Such systems are a "hart" of most accurate frequency standards: their frequency is locked to ultra-narrow clock transitions in atomic and ionic ensembles. Stability of optical clock on short averaging times (less than ensemble preparation time) is completely defined by clock laser. On longer times Dick effect, that is determined by level of laser noise, limits stability as well.

Basic technique to obtain ultra-low fractional instability is locking laser frequency to the mode of a passive monolith ultra-stable high-finesse Fabry-Perot cavity. It has to be vibroisolated and thermostabilized, is usually kept in ultra-high vacuum. Under those conditions fractional instability is fundamentally limited by thermal noise of cavity's mirrors (i. e. instability of length).

Allan variance of thermal noise $-\sigma_{y}-$ can be used to predict best achievable fractional instability of cavity's eigenmode. In dependency on major parameters it is given as:

$$
\sigma_{y} \propto \frac{\sqrt{T \times \phi_{\text {coat }}}}{L^{5 / 4} \times \lambda^{1 / 2} \times E^{1 / 2}}
$$

Here, $\mathrm{T}$ - temperature, $\phi$ coat - loss angle of mirror coating, $\mathrm{L}$ - cavity length, $\lambda$ - laser wavelength, E - Young's modulus of mirror's substrate.

Our main goal is to improve efficiency of ultra-stable laser systems, and, as a result, to provide laser systems with fractional frequency instability about $10^{-16}$ on 1 second averaging time. According to equation for $\sigma_{y}$, main ideas to diminish thermal noise influence are: using high-Q materials, low temperatures and enlarging base of reference cavity. In present work we consider four ultra-stable systems.

The idea of lowering temperature with stabilization at the point of zero extension is presented by two cryogenic single-crystal silicon cavities - one with dielectric $\mathrm{SiO}_{2} / \mathrm{Ta}_{2} \mathrm{O}_{5}$ mirrors (1) and one with crystalline GaAs/AlGaAs mirrors (2). Silicon is a very promising material for upcoming ultra-stable cavities because it has a temperature of zero extension at $124 \mathrm{~K}$ and has a very low mechanical loss angle. It is also transparent for $1.5 \mu \mathrm{m}$ wavelength,

\footnotetext{
* Corresponding author: denis.kryuchkov@phystech.edu
} 
which is covered by convenient erbium fiber lasers. Accurate calculations, proceeded for silica systems, resulted in following fundamental fractional instability limits: $3 \times 10^{-16}$ and $6 \times 10^{-17}$ for cavities with dielectric (1) and crystalline mirrors (2) respectively [2]. We have systems $(1,2)$ assembled at this moment. (1) has finesse about 580'000, measured by ringdown method; (3) - about 250'000. Both silica cavities are mounted in UHV cryogenic chambers and stabilized at $124 \mathrm{~K}$ with precision better than $3 \mathrm{mK}$. Fractional instability characterization via frequency correlation for two silica cavities will be performed in future.

The idea of enlarging cavity's base is presented by two large vertical and horizontal $(3,4)$ cavities made of Ultra-low Expansion glass, operating on $698 \mathrm{~nm}$ wavelength. These systems are being assembles at this moment. Fundamental frequency instability limit calculations resulted in $7 \times 10^{-17}$ for both large systems [2]. Also, vertical and horizontal cavities were numerically analyzed with finite element method to find out proper shape and optimal vibration-immune suspension configuration. Was found out that biconical is the best shape for (3): it affords least vibration susceptibility. For system (4) vibration-immune suspension system was developed, where bearings are located at the Airy points. Deformations under the action of horizontal and vertical accelerations were simulated for $(3,4)$ as well.

Presented systems $(3,4)$ will be used as clock lasers in $\mathrm{Sr}$ optical lattice clock in VNIIFTRI [3], systems $(1,2)$ - as optical frequency synthesizer stabilizers and for stabilizing long fiber-optic communication lines.

\section{References}

1. D. Matei et al., Phys. Rev. Lett. 118, 263202 (2017)

2. N. Zhadnov et al., Quantum Electronics, 48, 425 (2018)

3. O. Berdasov et al., Quantum Electronics, 47, 400 (2017) 